\title{
Fenoles simples de la destilación de Origanum vulgare valorados por espectrometría UV a través de auxocromos
}

\author{
Simple phenols from the distillation of Origanum vulgare assessed by UV \\ spectrometry through auxochromes
}

\author{
Johnny Daniel Bravo Loor'1, Francisco Alejandro Alcántara Boza², Angela Lorena Carreño Mendoza³, \\ Leonel Rolando Lucas Vidal ${ }^{4}$ Gustavo Adolfo Campuzano Marcillo ${ }^{5}$
}

Recibido: 13/07/2020 - Aprobado: 04/10/2021 - Publicado: 23/12/2021

\begin{abstract}
RESUMEN
Con la finalidad de determinar la cantidad de fenoles de un extracto oleoso de hojas de Origanum vulgare con un procedimiento ágil, accesible, económico y respetuoso con el entorno vital, se empleó la espectroscopia UV visible, la creación de un cromóforo a través de un auxocromo empleando cloruro de hierro III a $10000 \mathrm{mg} / \mathrm{L}$ en disolución de etanol 95\%, una amplitud de onda de 330 nanómetros y como referencia, un patrón de 5-Isopropil-2-metilfenol de 997000 mg/L de concentración, disueltos sucesivamente (9970 - 11000 - 12000 - 13000 - 14000 mg/L). La representación gráfica de comprobación de las cantidades conocidas de fenoles contenidas en las disoluciones y el producto de la extracción por arrastre de vapor de las hojas con respecto a la absorbancia, muestran luego del análisis estadístico de varianza por ajuste lineal de los mínimos cuadrados, una linealidad y precisión en el procedimiento utilizado, lográndose concentraciones entre 120000 y $140000 \mathrm{mg} / \mathrm{L}$ de componentes fenólicos como carvacrol. Se consiguió determinar la cantidad de estos polifenoles del Origanum vulgare, al establecer grupos insaturados y lograr densidades ópticas en el espectrofotómetro UV.
\end{abstract}

Palabras claves: Absorbancia; auxocromo; carvacrol; curva de calibración; ensayo FeCl3.

\begin{abstract}
In order to determine the amount of phenols in an oily extract of Origanum vulgare leaves with an agile, accessible, economical and respectful procedure with the living environment, UV visible spectroscopy was used, creating a chromophore with iron III chloride. at 10,000 mg / L in 95\% ethanol solution, a wave width of 330 nanometers and as a reference, a standard of 5-Isopropyl-2-methylphenol with a concentration of $997000 \mathrm{mg} / \mathrm{L}$, dissolved successively (99 $700-110000-120000-130000-140000 \mathrm{mg} / \mathrm{L})$. The graphical representation of verification of the known quantities of phenols contained in the solutions and the product of the extraction by vapor drag of the leaves with respect to the absorbance, shows after the statistical analysis of variance by linear adjustment of the least squares, a linearity and precision in the procedure used, achieving concentrations between 120,000 and $140,000 \mathrm{mg} / \mathrm{L}$ of phenolic components such as carvacrol. It was possible to determine the amount of these polyphenols from Origanum vulgare, by establishing unsaturated groups and achieving optical densities in the UV spectrophotometer.
\end{abstract}

Keywords: Absorbance; auxochrom; carvacrol; calibration curve; FeCl3 assay.

1 Universidad Nacional Mayor de San Marcos, Facultad de Ingeniería Geológica, Minera, Metalúrgica y Geográfica, Lima, Perú. Q.F. MPA. Doctorado en Ciencias Ambientales. Docente Escuela Superior Politécnica Agropecuaria de Manabí MFL, Ecuador.

Autor para correspondencia: jbravo@espam.edu.ec / jonisitobravol@hotmail.com

2 Universidad Nacional Mayor de San Marcos, Lima, Perú. Docente principal.

E-mail: falcantarab@unmsm.edu.pe - ORCID: https://orcid.org/0000-0001-9127-4450

3 Ingeniero PhD Docente. Universidad Técnica de Manabí, Ecuador.

E-mail: alcarreno@utm.edu.ec - ORCID: https://orcid.org/0000-0003-3370-7582

4 Ingeniero PhD. Escuela Superior Politécnica Agropecuaria de Manabí MFL, Ecuador.

E-mail: leonellucasvidal@espam.edu.ec- ORCID: https://orcid.org/0000-0002-2286-9407

5 Magister. Docente, Escuela Superior Politécnica Agropecuaria de Manabí MFL. Ecuador.

E-mail: gcampozanoregion4@gmail.com - ORCID: https://orcid.org/0000-0001-8969-2856 


\section{INTRODUCCIÓN}

Los extractos de Origanum vulgare nativos podrían resultar una opción como agentes que matan microorganismos o detiene su crecimiento, sin riesgos de originar tolerancias bacterianas ni repercusiones residuales, ya que el 5-isopropil-2-metilfenol y él 2-isopropil-5-metilfenol crean metabolitos los cuales no están directamente involucrados en el crecimiento, desarrollo o reproducción normal del organismo y se excretan en la micción $(90000 \mathrm{mg} / \mathrm{L})$ o se emiten por el sistema respiratorio $(100000 \mathrm{mg} / \mathrm{L})$ como anhidrido carbónico (Dusan Ninkov, 1999). Presentes en las plantas en su mayoría, los fenoles contienen grupos funcionales oxhidrilo sobre hidrocarburos aromáticos y coadyuvan a eludir padecimientos afines con problemáticas ocasionadas por tensiones oxidativas al actuar como antioxidantes (Huang et al., 2005). El líquido graso esencial de orégano mexicano (Lippia graveolens) inactivo el virus herpes simple-1 HSV-1 (cepas sensibles y tolerantes a medicamentos con acicloguanosina), virus sincitial respiratorio humano HRSV, herpesvirus bovino tipo 2 $\mathrm{BHV}-2$, anterior y ulterior a la infección del virus, mientras que el 5-isopropil-2-metilfenol (carvacrol) ocasionó la inactivación posterior a introducir estos acelulares segmentos de ARN (Pilau et al., 2011). En los líquidos oleosos de Origanum vulgare preponderan los componentes polifenólicos, para la ciencia que estudia la composición y reacciones químicas en los seres vivos a los compuestos fenólicos nativos que se encuentran en la mayoría de las plantas lo relacionan con la función hidroxilo (Khoddami et al., 2013), (Amorati \& Valgimigli, 2012), (Robbins, 2003). Así, reportes de investigaciones indican su actividad antiinflamatoria y antibacteriana (Dos Santos et al., 2006); no obstante, las técnicas para ponderarlos y comprobarlos resultan caros cuando se utilizan procedimientos por cromatografía líquida de alta resolución y gaseosa. La cromatografía líquida tiene aplicaciones, elevados costos y el uso de solventes no amigables con el ambiente (Gomis Yagüez, 2008). Pero también presentan limitaciones por la naturaleza de la muestra influyendo en la selectividad, la sensibilidad y la resolución (Marriott et al., 2001). Así mismo limitaciones que pueden tener estas cromatografías como que el compuesto debe ser térmicamente estable y volátil, entonces, la cromatografía de gases se emplea cuando los componentes de la mezcla problema son volátiles o semivolátiles y térmicamente estables a temperaturas de hasta $350-400^{\circ} \mathrm{C}$ (Malviya $\mathrm{R}$ et al., 2010). Además, la utilización de los solventes para HPLC como metanol, acetonitrilo, agua o sus mezclas deben tener grado reactivo de mucha pureza. Otro de los métodos de cuantificación para aceites esenciales se efectúa con equipos menos sofisticados, un poco más accesible y respetuoso con el entorno vital, el espectrofotómetro UV visible, la cual ha sido uno de los que más ampliamente se ha empleado por su simplicidad y bajo precio (Gutiérrez Gaitén et al., 2000), para el cual se usan procedimientos como el de FolinCiocalteu que contiene sales del ion molibdeno y tungsteno de sodio y, reaccionan con cualquier espécimen de ácido fenico, creando compuestos coordinados fosfomolíbdicosfosfotúngsticos (Peterson, 1979), formándose un cromógeno de coloración azulada activa, proporcional a la cantidad de grupos oxhidrilo presente en los elementos. Los ensayos se crearon inicialmente en la determinación de L. tirosina en polipéptidos (Singleton V L \& Rossi J A Jr., 1965), pero con los años fue rectificado para determinar componentes fenólicos en diferentes extractos de plantas (Singleton et al., 1999). Numerosos componentes químicos no absorbentes se pueden establecer al reaccionar con sustancias cromóforas, creando combinados con absorción muy activa en las regiones UV-VIS, esta es la parte de una molécula responsable de su color (IUPAC, 2019). El espectro de absorción de un elemento orgánico resulta de las interrelaciones de las partículas elementales de la luz y las partículas subatómicas que contribuye claramente en la creación de las uniones, y por tanto se relacionan con más de una partícula o con aquellos que se sitúan cerca de partículas atómicas como $\mathrm{O}, \mathrm{S}, \mathrm{N}$ y los halógenos (Skoog D et al., 2001). Los compuestos fenólicos son muy reactantes a la renovación aromatizada electrofílica por el grupo oxhidrilo enérgicamente reactivo. Al reaccionar con el tricloruro de hierro $\left(\mathrm{FeCl}_{3}\right)$, el Fe funcionara a modo de ion y al reactivarse con oxhidrilos de combinaciones aromáticas crea complejos con características de grupos insaturados que absorben más espectro de luz, un auxocromo sustituye y se fija al cromóforo y aumenta la radiación y la $\lambda$ (longitud de onda) de la absorción, (Pavia et al., 2012) (ver Figura 1)

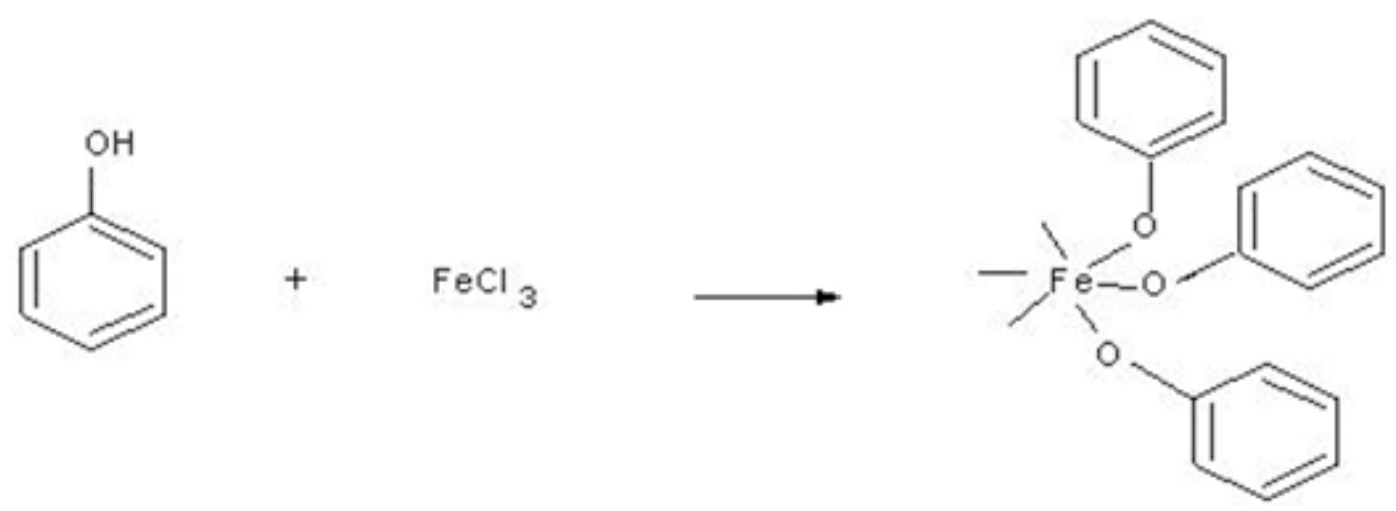

Figura 1. La interacción para crear un combinado de coordinación (cromóforo), el ion Férrico reacciona con grupos oxhidrilos (Pavia et al., 2012). 
Generalmente estos compuestos no presentan absorción a radiaciones vislumbradas en el rango UV-visible y se plantea, establecer transiciones electrónicas trazables en la generación de combinados de grupos iónicos o cromóforos para cuantificar su absorción y, aplicando curvas de comprobación establecer la cantidad de elementos poli fenólicos en un espécimen de extracto oleoso de orégano ¿empleando la espectrofotometría ultravioleta visible?

\section{MÉTODOS}

\subsection{Extracción y determinación de compuestos} fenólicos por espectrofotometría UV visible

Se equipó en el laboratorio un destilador de vidrio articulado a un calorífero para generar calor, para condensar un refrigerante y un colector de agua como condensador.

Para la extracción del aceite acuoso de las hojas se recolectó el orégano del ambiente, lavó y se destilo al vapor, el extracto obtenido se separó de la mayor cantidad de agua con una ampolla de separación (Guenther, 1972).

Se utilizó como referencia un patrón de 5-isopropil-2metilfenol concentración 997000 mg/L, de la fábrica Merck, los análisis fueron efectuados en un espectrofotómetro UVvisible Jenway 736-501. Los ensayos se efectuaron luego de un barrido espectral a una longitud de onda de 320 nanómetros.

Se utilizaron micropipetas graduadas de $0.005 \mathrm{ml}$ $0.2 \mathrm{ml}$ y $0.1 \mathrm{ml}-1.0 \mathrm{ml}$, peras de decantación de $250 \mathrm{ml}$, fiolas Erlenmeyer de $1000 \mathrm{ml}$, balón de destilación de $500 \mathrm{ml}$, fiolas aforadas de $10 \mathrm{ml}$, tubos cilíndricos de vidrio para doblar y recipientes de cuarzo para el espectrofotómetro.

\subsection{Elaboración de patrones}

A partir del patrón de referencia 5-isopropil-2-metilfenol $997000 \mathrm{mg} / \mathrm{L}$ de concentración, se disolvieron con alcohol etílico al $95 \% \mathrm{v} / \mathrm{v}$ una serie de 5 soluciones estandarizadas (99 700 - 110000 - 120000 - 130000 - 140000 mg/L v/v).

Para el efecto batacrómico del cromóforo se formó un auxocromo de $\mathrm{FeCl}_{3}$ al $1 \% \mathrm{p} / \mathrm{v}$ en disolución de alcohol etílico de $95 \% \mathrm{p} / \mathrm{v}$. La prueba de cloruro de hierro III se usó para comprobar y cuantificar polifenoles en el espécimen o componente, como fenol natural del extracto de plantas de orégano. En la cubeta de cuarzo se adiciono $500 u$ de alcohol etílico de 97\%, el extracto de Origanum vulgare (10ul), cloruro de hierro III al 1\% (10ul), y se dejó reposando por cinco minutos.

Se efectuaron variaciones en la longitud de onda, con la finalidad de encontrar la máxima absorbancia en el rango de 200-500 nanómetros del cromógeno (extracto oleoso de Origanum vulgare $+\mathrm{FeCl}_{3}$ ) y como blanco, cloruro de hierro III al 1\% en alcohol etílico. Se estableció una longitud de $320 \mathrm{~nm}$ y se lee las absorbancias de los compuestos que contienen los patrones elaborados a partir del estándar de referencia y las muestras.

Para efectuar el tratamiento de los mínimos cuadrados, se realizó una curva de calibración, esta es la representación gráfica de una señal (absorbancia), que se mide en función de la concentración de un analito (Harris, 2016), consideradas variables en estudio.

\subsection{Análisis estadístico}

Se utilizó un ANOVA con el método estadístico de regresión de los mínimos cuadrados y obtener excelente precisión y confianza estadística en las cuantificaciones de la curva y, que esta recta de calibrado haga que la suma de los cuadrados de las distancias verticales entre cada punto experimental y la recta de calibrado sea mínima o tienda a cero. Se utilizo para mantener el registro el Sistema de Análisis Estadístico SAS y se le añadió las variables concentración y absorbancia con un total de 45 replicaciones.

En la tabla 1 se observa valores del coeficiente de determinación (0.5203) y el coeficiente de determinación ajustado (0.5091) con bajo sesgo, indicando que el coeficiente de determinación que se obtuvo es relativamente aceptable para determinar el ajuste de la curva.

Tabla 1. Método REG Modalidad - Modelo 2 Variable dependiente ly

\begin{tabular}{|c|c|c|c|c|c|c|c|}
\hline & \multicolumn{5}{|c|}{ Cantidad de informaciones leídas } & 45 & \\
\hline & \multicolumn{5}{|c|}{ Cantidad de lecturas usadas } & 45 & \\
\hline \multicolumn{8}{|c|}{ ANOVA } \\
\hline \multicolumn{2}{|c|}{ Origen } & DF & $\begin{array}{l}\text { Suma de } \\
\text { cuadrados }\end{array}$ & \multicolumn{2}{|c|}{$\begin{array}{l}\text { Cuadrado } \\
\text { de la media }\end{array}$} & F-Valor & $\mathrm{Pr}>\mathrm{F}$ \\
\hline Patrón & & 1 & 29.58879 & & 58879 & 6.64 & $<.0001$ \\
\hline Error & & 43 & 27.28029 & & 3443 & & \\
\hline \multicolumn{2}{|c|}{ Total, corregido } & 44 & 56.86907 & & & & \\
\hline & \multicolumn{3}{|c|}{ Raíz MSE } & 0.79651 & 51 R-cuadrado & 0.5203 & \\
\hline & \multicolumn{3}{|c|}{ Media dependiente } & -2.04418 & 18 R-Sq Ajust & 0.5091 & \\
\hline & \multicolumn{3}{|c|}{ Coef Var } & -38.96475 & & & \\
\hline \multicolumn{8}{|c|}{ Tasadores de parámetros } \\
\hline Variable & DF & Tasad & lor del pará & ametro & Error estándar & Valor $\mathrm{t}$ & $\operatorname{Pr}>|t|$ \\
\hline Intercept & 1 & & & .88015 & 1.00800 & -8.81 & $<.0001$ \\
\hline$x$ & 1 & & & .56995 & 0.08346 & 6.83 & $<.0001$ \\
\hline
\end{tabular}

\section{RESULTADOS}

El extracto de orégano obtenido alcanzó óptimas densidades ópticas en el espectroscopio ultravioleta visible a una amplitud de onda de $320 \mathrm{~nm}$. con la creación de un cromóforo al reaccionar con $\mathrm{FeCl}_{3}$ al $1 \%$ en etanol de $95 \%$. Se determinó las concentraciones de compuestos fenólicos como carvacrol empleando a la variable dependiente absorbancia, factores de concentración de la muestra y la serie de estándares en el análisis de la varianza. En la figura 2 se observa que el valor $\mathrm{R}^{2}$ de $95 \%$ se ajusta al modelo lineal de datos en la curva de calibración con el valor de $\mathrm{R}^{2}$ ajustado como buen predictor en las determinaciones.

En la figura 3 se observa el proceder de la variable dependiente Y (absorbancia) en relación con la variable $\mathrm{X}$ (concentración) en los valores de regresión lineal. Los coeficientes de determinación oscilan entre 0 y 1 para un mejor ajuste entre los valores observados y los valores de predicción del modelo, estas son pequeñas y no presentan sesgo de la variable en estudio. 


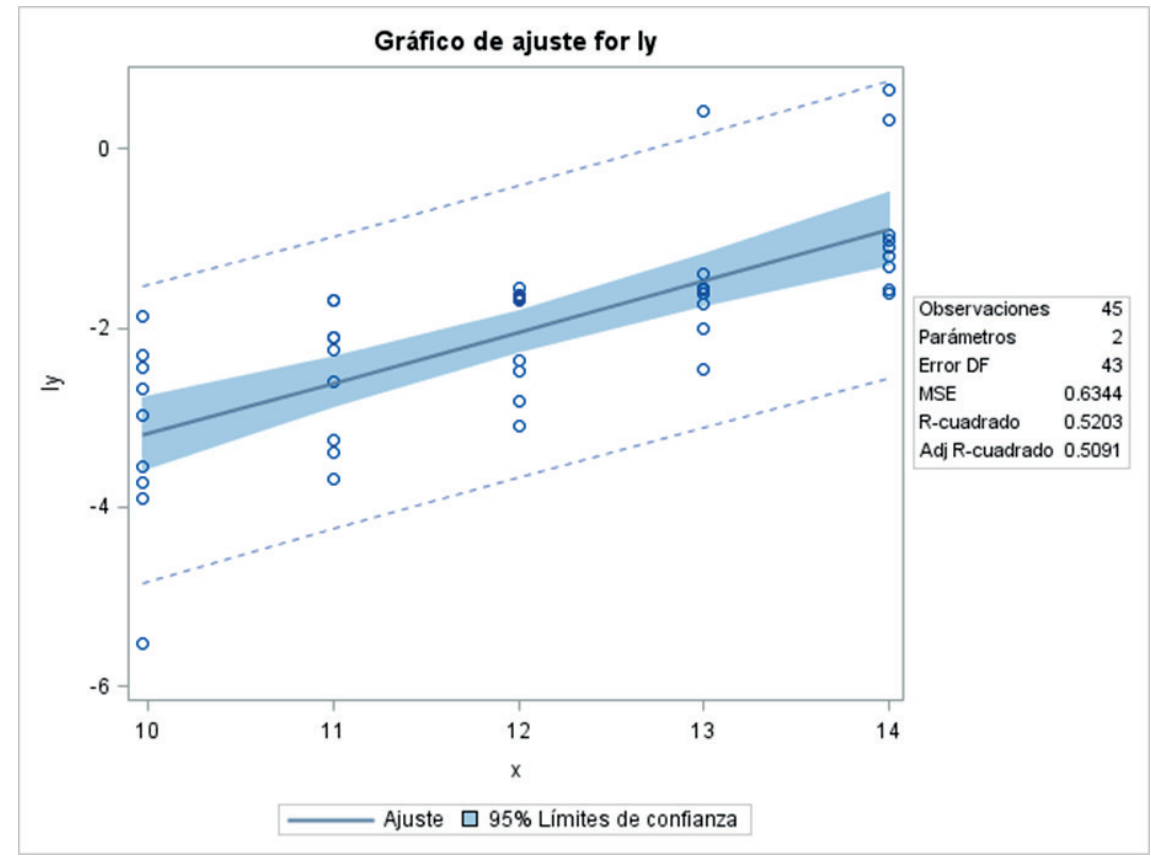

Figura 2. Gráfico de comprobación con $n=5$ para la cuantificación de los estándares y especímenes frente a las absorbancias.

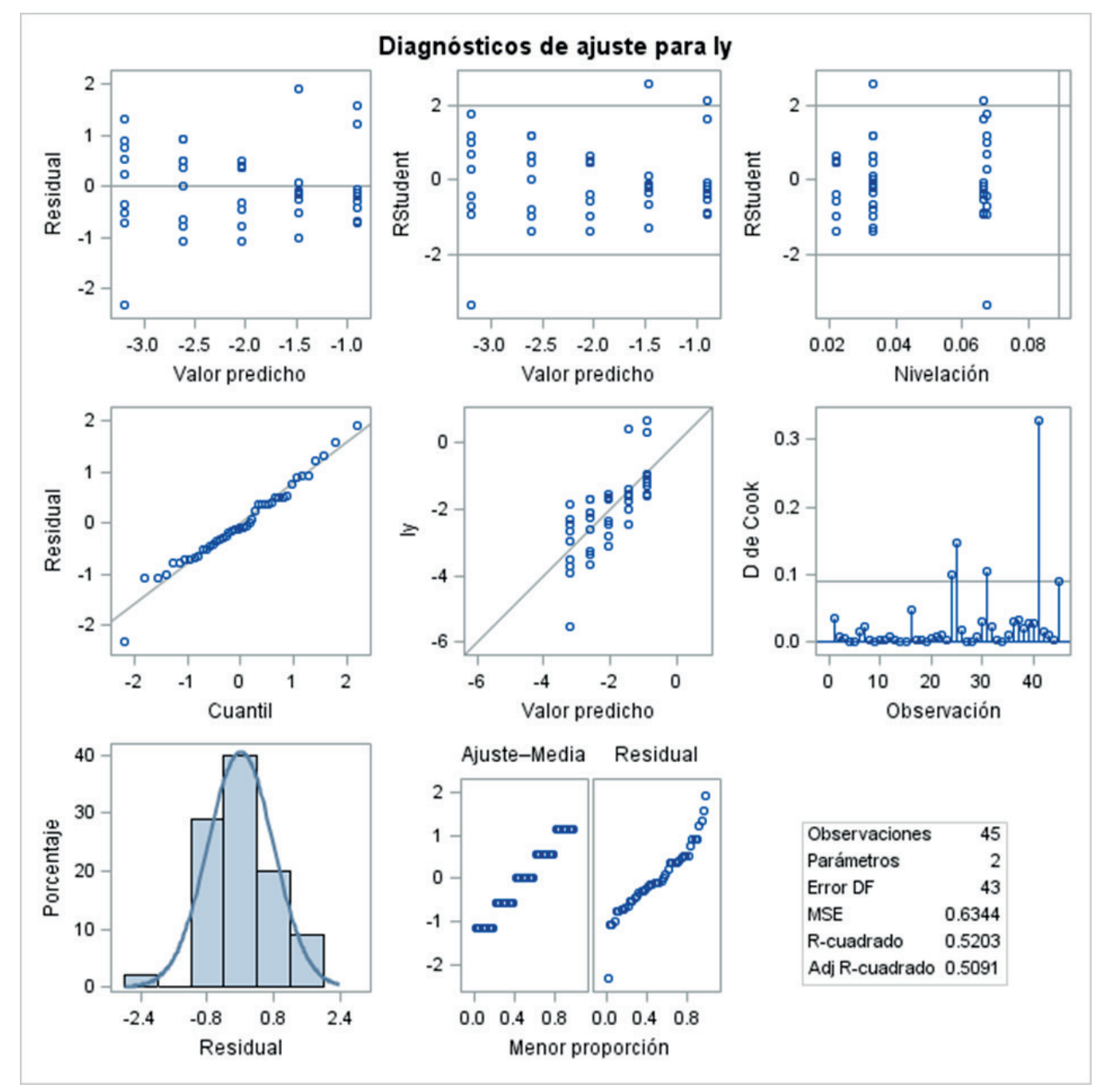

Figura 3. Análisis de ly de los arreglos de media y residual de las 45 lecturas. 
Los valores de la tabla 2 de las absorciones leídas en el espectroscopio UV visible. La izquierda las absorbancias y a la derecha, resultados de los contenidos de muestras del extracto oleoso de orégano y los estándares que presentan contenidos entre 12 y $14 \%$ de compuestos poli fenólicos.

Tabla 2. Muestra resultados de absorbancias y concentración de estándares y muestras

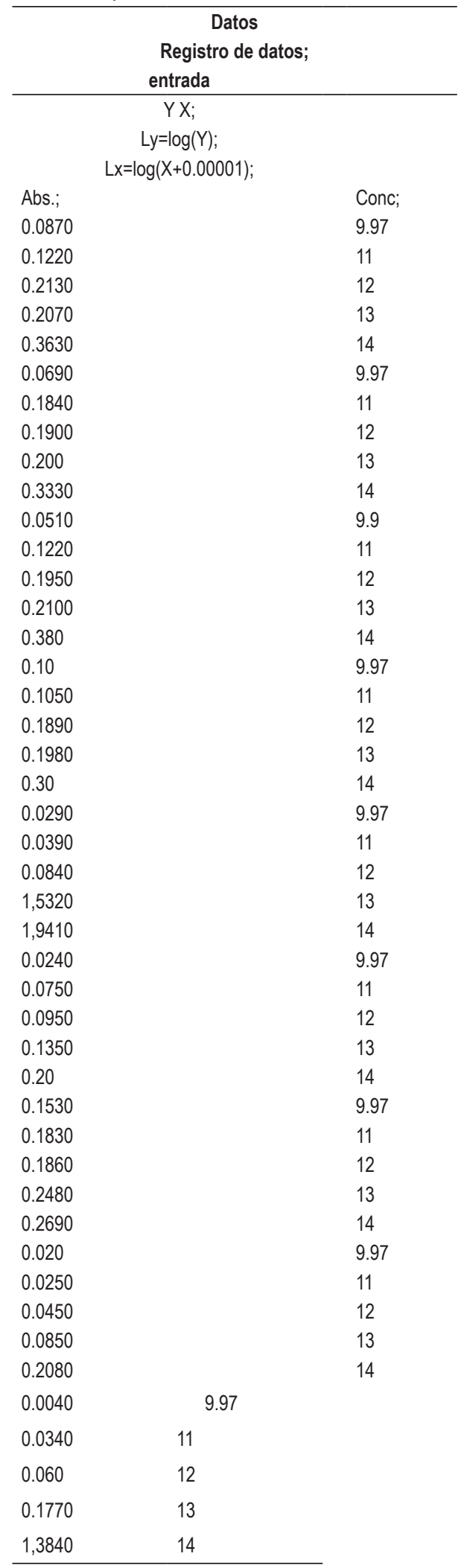

En la Figura 4 se aprecia la línea de regresión en azul muestra el mejor ajuste y los interceptó con resultados.

\section{DISCUSIÓN}

Las funciones oxhidrilos de fenoles líquidos oleosos que intervienen como grupos positivos de átomos y el $\mathrm{FeCl}_{3}$, establecen un enlace coordinado e ion metálico y/o molécula responsable del color, un grupo químico idóneo para sorber luminiscencia selectivamente, resultando de la tonalidad de ciertas mezclas orgánicas, encargadas de originar coloración (Allen \& Koumanova, 2005), (Gupta \& Suhas, 2009). Las características del disolvente, potencial de $\mathrm{H}$ de la solución, nivel térmico, cantidad de minerales iónicos y la existencia de elementos que interfieran, pueden trascender en los espectros de absorción de las sustancias, así como diferenciaciones en el ancho de la hendidura (amplio de franja seguro) en el espectrómetro (Gutiérrez Gaitén et al., 2000).

Estos métodos para comprobar compuestos polifenólicos son simples y de bajo precio, pero solo dan una apreciación de las concentraciones de estos por arriba de cierto nivel y no los ponderan específicamente; no obstante, estas técnicas logran ser ventajosas para la detección rápida y correspondientemente económica en muchos especímenes (Ignat et al., 2011), así mismo coincide, que a pesar de que hay un sinnúmero de estudios publicados, reiterarlos no dejará de ser importante. La cuantificación estructural de varios grupos fenólicos sigue siendo difícil (Tsao \& Yang, 2003). Así, hay un elevado margen para fomentar técnicas de medición establecidos por el tipo de grupo polifenólico (Liu et al., 2011).

En ensayos espectrofotométricos UV los métodos de Folin-Denis y Folin-Ciocalteu son muy empleados en la determinación de la concentración de compuestos fenólicos en materias vegetales desde hace muchos años (Lapornik et al., 2005). En el uno y el otro método los electrones son ganados para reducir su valencia por contener tungsteno y molibdeno (Stalikas, 2007). No obstante, estos productos vegetales pudiesen contener una gran cantidad de compuestos con particularidades no poli fenólicas que incluyen glúcidos de tipo reductor como glucosa y fructosa, moléculas orgánicas con grupos amino y carboxilo y, ácidos ascórbicos, (Granato et al., 2016), (Lester et al., 2012), (Ludwig et al., 2013) y (Shanmugavelan et al., 2013), que podrían interferir con la determinación de estos compuestos.

La cuantificación propuesta obtuvo entre 12 y $14 \%$ de componentes fenólicos como carvacrol, relativamente bajas debido al procedimiento de extracción en las hojas de orégano. La cuantificación de compuestos fenólicos en muestras vegetales, está muy asociada a los métodos utilizados para la extracción (Tura \& Robards, 2002).

\section{CONCLUSIONES}

El extracto de orégano puro obtenido por destilación al vapor al crearle cromóforos, grupos no saturados de compuestos coordinados de color amarillo verdoso, consiguió absorber 


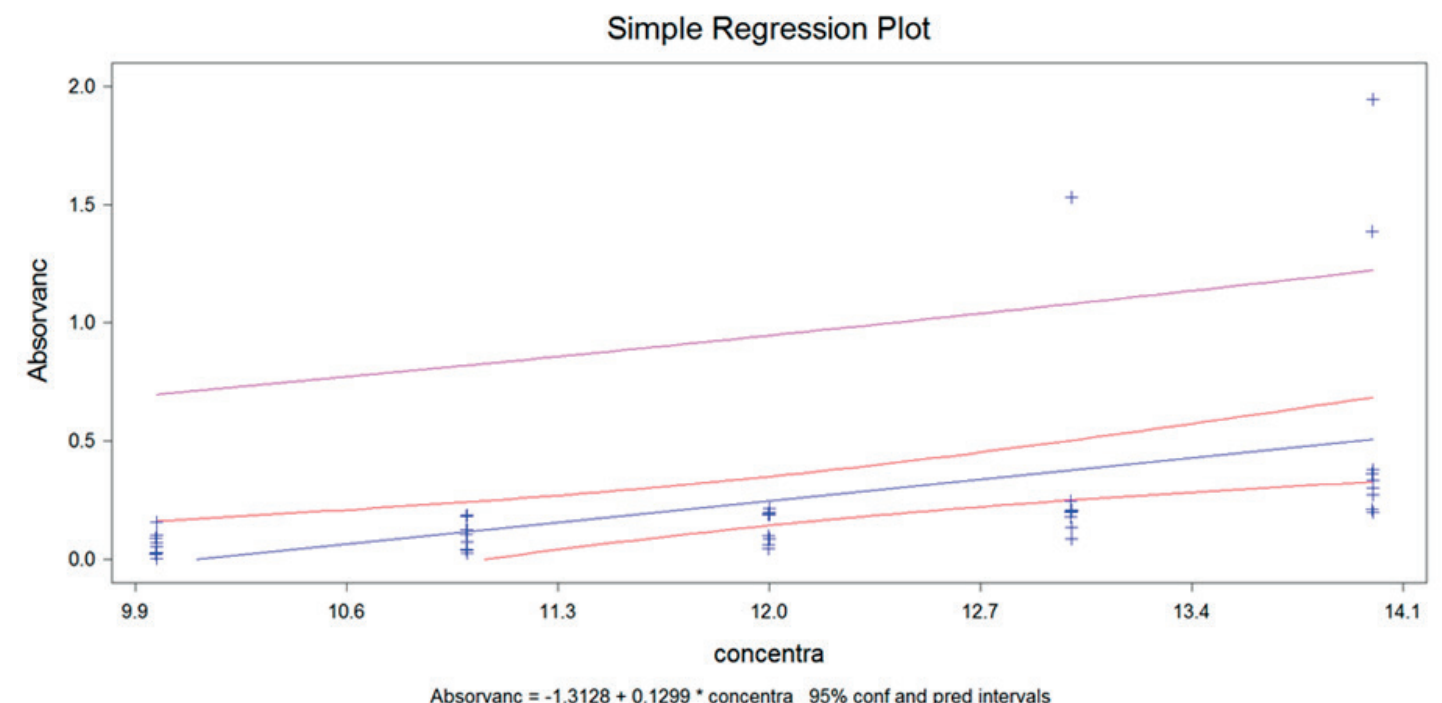

Figura 4. Modelo lineal de los mínimos cuadrados en función de la absorbancia y concentración.

radiación en el espectrofotómetro UV visible y contribuyó para la valoración de compuestos fenólicos como carvacrol, con concentraciones entre 12 y $14 \%$.

\section{AGRADECIMIENTOS}

Agradezco a la FIGMMG-UNMSM de Lima Perú y a la carrera de Medicina veterinaria ESPAM MFL de Calceta Ecuador.

\section{REFERENCIAS}

Allen, S. J., \& Koumanova, B. (2005). Decolourisation of water/ wastewaterusing adsorption. Journal of the University of Chemical Technology and Metallurgy, 40, 3. https://dl.uctm. edu/journal/node/j2005-3/Revew.pdf

Amorati, R., \& Valgimigli, L. (2012). Modulation of the antioxidant activity of phenols by non-covalent interactions. In Organic and Biomolecular Chemistry (Vol. 10, Issue 21, pp. 4147-4158). https://doi.org/10.1039/c2ob25174d

Dos Santos, M. D., Almeida, M. C., Lopes, N. P., \& De Souza, G. E. P. (2006). Evaluation of the anti-inflammatory, analgesic and antipyretic activities of the natural polyphenol chlorogenic acid. Biological and Pharmaceutical Bulletin, 29(11), 2236-2240. https://doi.org/10.1248/bpb.29.2236

Dusan Ninkov. (1999). Pharmaceutical compositions suitable for use against histomoniasis (Patent No. US5990178A).

Gomis Yagüez, V. (2008). Cromatografía de líquidos de alta resolución. Universidad de Alicante.

Granato, D., Santos, J. S., Maciel, L. G., \& Nunes, D. S. (2016). Chemical perspective and criticism on selected analytical methods used to estimate the total content of phenolic compounds in food matrices. In TrAC - Trends in Analytical Chemistry (Vol. 80, pp. 266-279). https://doi.org/10.1016/j. trac.2016.03.010
Guenther, E. (1972). The Essential Oils: History, Origin in Plants, Production. Analysis, 1, 147-151.

Gupta, V. K., \& Suhas. (2009). Application of low-cost adsorbents for dye removal - A review. In Journal of Environmental Management (Vol. 90, Issue 8, pp. 2313-2342). https://doi. org/10.1016/j.jenvman.2008.11.017

Gutiérrez Gaitén, Y. I., Miranda Martínez, M., Varona Torres, N., \& Rodríguez, A. T. (2000). Validación de dos métodos espectrofotométricos para la cuantificación de taninos y flavonoides (quercetina) en Psidium guajaba, L. Revista Cubana de Farmacia, 34(1), 50-55.

Harris, D. C. (2016). Analisis quimico cuantitativo (3a. ed.).

Huang, D., Boxin, O. U., \& Prior, R. L. (2005). The chemistry behind antioxidant capacity assays. In Journal of Agricultural and Food Chemistry (Vol. 53, Issue 6, pp. 1841-1856). https://doi.org/10.1021/jf030723c

Ignat, I., Volf, I., \& Popa, V. I. (2011). A critical review of methods for characterisation of polyphenolic compounds in fruits and vegetables. In Food Chemistry (Vol. 126, Issue 4, pp. 1821 1835). https://doi.org/10.1016/j.foodchem.2010.12.026

IUPAC. (2019). The IUPAC Compendium of Chemical Terminology. In The IUPAC Compendium of Chemical Terminology. https://doi.org/10.1351/goldbook

Khoddami, A., Wilkes, M. A., \& Roberts, T. H. (2013). Techniques for analysis of plant phenolic compounds. In Molecules (Vol. 18, Issue 2, pp. 2328-2375). https://doi.org/10.3390/ molecules 18022328

Lapornik, B., Prošek, M., \& Wondra, A. G. (2005). Comparison of extracts prepared from plant by-products using different solvents and extraction time. Journal of Food Engineering, 71(2), 214-222. https://doi.org/10.1016/j. jfoodeng.2004.10.036

Lester, G. E., Lewers, K. S., Medina, M. B., \& Saftner, R. A. (2012). Comparative analysis of strawberry total phenolics via Fast Blue BB vs. Folin-Ciocalteu: Assay interference by 
ascorbic acid. Journal of Food Composition and Analysis, 27(1), 102-107. https://doi.org/10.1016/j.jfca.2012.05.003

Liu, P., Kallio, H., \& Yang, B. (2011). Phenolic compounds in hawthorn (Crataegus grayana) fruits and leaves and changes during fruit ripening. Journal of Agricultural and Food Chemistry, 59(20), 11141-11149. https://doi.org/10.1021/ jf202465u

Ludwig, I. A., Bravo, J., De Peña, M. P., \& Cid, C. (2013). Effect of sugar addition (torrefacto) during roasting process on antioxidant capacity and phenolics of coffee. $L W T$ - Food Science and Technology, 51(2), 553-559. https://doi org/10.1016/j.lwt.2012.12.010

Malviya R, Bansal V, Pal O.P., \& Sharma P.K. (2010). High performance liquid chromatography: a short review. Journal of Global Pharma Technology. https://www.researchgate.net/ profile/Rishabha-Malviya/publication/235987484 High performance_liquid_chromatography_A_short_review/ links/02e 7e5153e680408ac000000/High-performanceliquid-chromatography-A-short-review.pdf

Marriott, P. J., Shellie, R., \& Cornwell, C. (2001). Gas chromatographic technologies for the analysis of essential oils. In Journal of Chromatography A (Vol. 936, Issues 1-2, pp. 1-22). https://doi.org/10.1016/S0021-9673(01)01314-0

Pavia, D., Kriz, G., Engel, R., \& Lampman, G. (2012). A Microscale Approach to Organic Laboratory Techniques. Brooks/Cole; 5th Edition, 1-1040.

Peterson, G. L. (1979). Review of the folin phenol protein quantitation method of lowry, rosebrough, farr and randall In Analytical Biochemistry (Vol. 100, Issue 2, pp. 201-220). https://doi.org/10.1016/0003-2697(79)90222-7

Pilau, M. R., Alves, S. H., Weiblen, R., Arenhart, S., Cueto, A. P., \& Lovato, L. T. (2011). Antiviral activity of the Lippia graveolens (Mexican oregano) essential oil and its main compound carvacrol against human and animal viruses. Brazilian Journal of Microbiology, 42(4), 1616-1624. https://doi.org/10.1590/S1517-83822011000400049
Robbins, R. J. (2003). Phenolic acids in foods: An overview of analytical methodology. In Journal of Agricultural and Food Chemistry (Vol. 51, Issue 10, pp. 2866-2887). https:// doi.org/10.1021/jf026182t

Shanmugavelan, P., Kim, S. Y., Kim, J. B., Kim, H. W., Cho, S. M., Kim, S. N., Kim, S. Y., Cho, Y. S., \& Kim, H. R. (2013). Evaluation of sugar content and composition in commonly consumed Korean vegetables, fruits, cereals, seed plants, and leaves by HPLC-ELSD. Carbohydrate Research, 380, 112-117. https://doi.org/10.1016/j.carres.2013.06.024

Singleton, V. L., Orthofer, R., \& Lamuela-Raventós, R. M. (1999). Analysis of total phenols and other oxidation substrates and antioxidants by means of folin-ciocalteu reagent. Methods in Enzymology, 299, 152-178. https://doi.org/10.1016/ S0076-6879(99)99017-1

Singleton V L, \& Rossi J A Jr. (1965). Colorimetry of total phenolics with phosphomolybdic-phosphotungstic acid reagents. American Journal of Enology and Viticulture, 16(3), 144-158.

Skoog D, Holler F, \& Nieman M. (2001). Principios de Análisis Instrumental. McGraw-Hill (5ta Edición).

Stalikas, C. D. (2007). Extraction, separation, and detection methods for phenolic acids and flavonoids. In Journal of Separation Science (Vol. 30, Issue 18, pp. 3268-3295). https://doi.org/10.1002/jssc.200700261

Tsao, R., \& Yang, R. (2003). Optimization of a new mobile phase to know the complex and real polyphenolic composition: Towards a total phenolic index using high-performance liquid chromatography. Journal of Chromatography A, 1018(1), 29-40. https://doi.org/10.1016/j.chroma.2003.08.034

Tura, D., \& Robards, K. (2002). Sample handling strategies for the determination of biophenols in food and plants. In Journal of Chromatography A (Vol. 975, Issue 1, pp. 71-93). https:// doi.org/10.1016/S0021-9673(02)00879-8 